\title{
Lipid Translocation (Flip-Flop) As One of the Least Understood Dynamical Processes in Articular Cartilage Membrane
}

\section{Zenon Pawlak}

Tribochemistry Consulting, Salt Lake City, Utah 84117, USA and University of Economy, Biotribology Laboratory, 85-229 Bydgoszcz, Poland

Corresponding author: Zenon Pawlak, Tribochemistry Consulting, Salt Lake City, Utah 84117, USA and University of Economy, Biotribology Laboratory, 85-229 Bydgoszcz, Poland.

Received date: April 06, 2020; Accepted date: June 12, 2020; published date: July 10, 2020

Citation: Zenon P, Lipid Translocation (Flip-Flop) As One of the Least Understood Dynamical Processes in Articular Cartilage Membrane. J Clinical Research and Reports, 4(5); DOI:10.31579/2690-1919/087

Copyright: ( 2020 Zenon P. This is an open access article distributed under the Creative Commons Attribution License, which permits unrestricted use, distribution, and reproduction in any medium, provided the original work is properly cited.

\begin{abstract}
The lipids molecules translocation in phospholipid membrane is named the (flip-flop) event. For this purpose, the translocation may take place in vivo with the surface of articular cartilage. Flip-flops are one of the least understood dynamical processes in vivo in cartilage membranes. In this work, we explain the molecular mechanism lipid translocation (flip-flop) in vivo during the drying process cartilage surface. Wettability of cartilage surface vs. drying time was determined. Our findings strongly support the idea that the process of translocation lipid (flip-flop) transformed hydrophilic surface in hydrophobic in dry-air condition for healthy and osteoarthritic cartilage. Such material can be classified cartilage as "smart material" and this fact is poorly known in the literature.
\end{abstract}

Keywords: Transformation flip-flop; Cartilage; Wettability; Hydrophilic; Hydrophobic

\section{Introduction}

Phospholipids molecules in aqueous media spontaneously arrange into a bilayer on the surface of articular cartilage. The bilayer is held together by hydrophobic interactions between the tails. Phospholipid (PL) bilayers on the surface of articular cartilage provide characteristics that are well adapted to wet and relatively dry conditions. This smart surface characteristic creates a hydrophobic-hydrophilic balance resulting in a functional hydrophilic surface in the intact joint. One of the quantitative indicators of surface tribochemical properties is wettability. This is measured as the contact angle between a drop of water and the reference surface.

Wettability characterizes the surface of various objects, which are generally defined as wettable (highly hydrophilic, $\theta \sim 0^{\circ}$ to $45^{\circ}$ ) or non-wettable (highly hydrophobic, $\theta \sim 90^{\circ}$ to $180^{\circ}$ ).
The mechanisms to actively transport lipids across a lipid bilayer using specific membrane are widely recognized [1]. All lipids in biomembranes can move within the bilayer, allowing for membrane fluidity and flexibility. Exchanging places with their neighbors by slow transverse diffusion (see Fig. 1) and fast lateral shift $\left(\sim 10^{-6} \mathrm{sec}\right)$. Double bonds in unsaturated hydrocarbon chains also tend to increase fluidity of the phospholipid bilayers. This fluidity allows for the spontaneous breaking and reforming of membranes [1,2]. This is called flip-flop mechanism. Membrane fluidity is enhanced at higher temperatures and is also affected by the composition of the bilayer. The formation of a transient water pore (defect) in the membrane inevitably leads to diffusive translocation of lipids through the pore, which is driven by thermal fluctuations (Fig.1] [3].

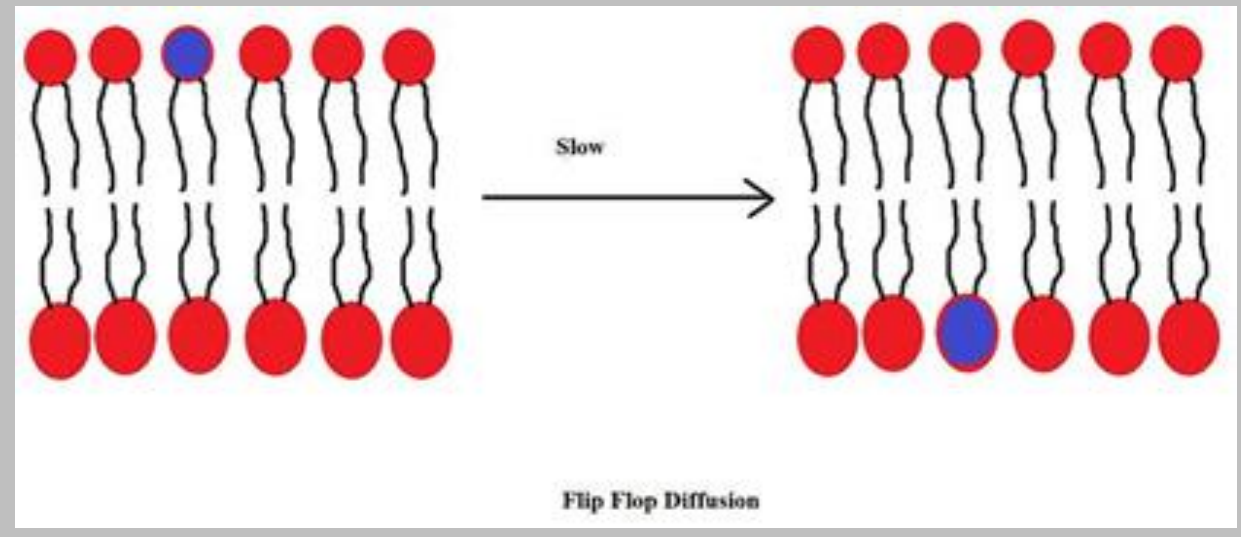

Fig.1. The motion of phospholipid with lipid bilayer in aqueous solution. Transverse diffusion (flip-flop) is slow $\left(\sim 10^{5} \mathrm{sec}\right)$. 
The experimentally observed fact that the exposure of lipid membranes to electric field pulses considerably reduces the time required for lipid flipflops. Negatively charged surface of articular cartilage membrane inevitably leads to diffusive translocation of lipid flip-flop.

In this work, wettability of cartilage surface vs. drying time was determined. Wettability helps us to explain the molecular mechanism lipid translocation (flip-flop) for healthy and osteoarthritic cartilage. Lipid translocation in cartilage membranes is still far from being understood and characterized. It is observed that lipid flip-flop is affected by dehydration activated process. Moreover, we report for the first time wettability of unhealthy cartilage in vivo changes own characteristics.

\section{Experimental}

In the delipidization procedure a Folch reagent $(2: 1 \mathrm{v} / \mathrm{v}$ mixture of chloroform and methanol) was used to gradually remove the PL bilayers from the bovine cartilage surface. The samples were immersed in the reagent mixture for 3,9 and 19 minutes at the same meniscus. After extraction, the sample was placed in saline solution for 1 hour to remove the residual solvent and promote rehydration and then air-dried. These samples were used for the surface wettability and friction measurements. The delipidization procedure removed most of the PL, although some amount of a hydrophobic proteolipid remained as a minor component [4].

Contact angle measurements The contact angle between the liquid and the tested cartilage was measured using a KSV CAM100 tensiometer. A tensiometer is a device for measuring the surface tension of a liquid. The contact angle measured was that between a droplet of a $0.15 \mathrm{M}$ saline solution and a given air-dry cartilage surface. The contact angle measurements of the normal (intact) and the delipidated cartilage samples were carried out under dry-air atmosphere in the laboratory $[5,6]$ at ambient temperature of $22^{\circ} \mathrm{C}$ and a relative humidity (HR) of approximately $45 \%$. Five tests were performed on each specimen and each set-up. The contact angle test performed on the normal, partial and completely depleted cartilage samples. The depleted cartilage samples imitate osteoarthritic degradation of cartilage surface.

\section{Results and discussion}

Formation of structural defects (water pore) is the result of an increase in the lateral surface tension induced by the evaporation surface water. This surface tension produced by formation of a transient water dehydration in the membrane inevitably leads to diffusive translocation of lipids (flipflop) can in turn induce changes from bilayer to monolayer (Fig. 2). A change in surface energy leads to conformational changes in the surface of bovine patella from bilayer (super hydrophilic $\sim 0^{\circ}$ contact angle) to monolayer (hydrophobic $\sim 104^{\circ}$ ). Changes of the wettability contact angle as a function of air-drying time are shown in Fig.2 (c) with totally depleted surface of phospholipid bilayer curve (1) contact angle of $40^{\circ}$; partially depleted PL curve (2) and (3) contact angle of $63^{\circ}$, and $75^{\circ}$, and curve (4) normal articular surface; contact angle of $104^{\circ}$.
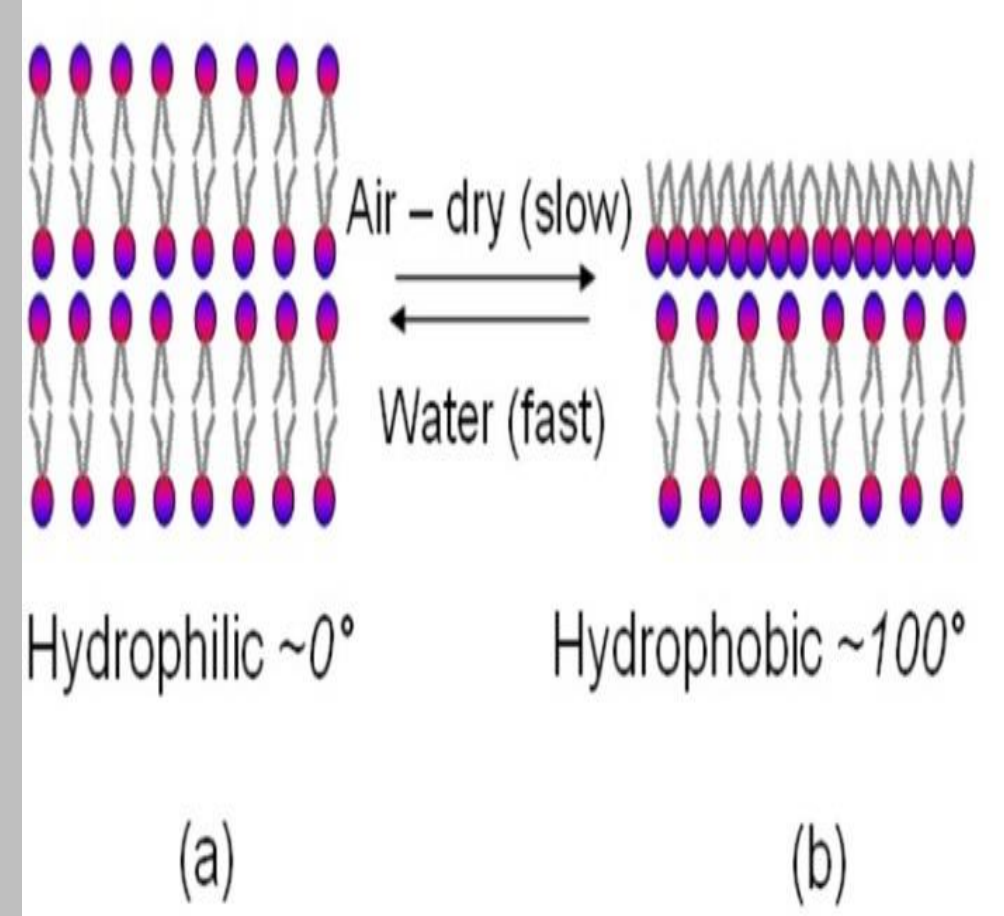

(b)

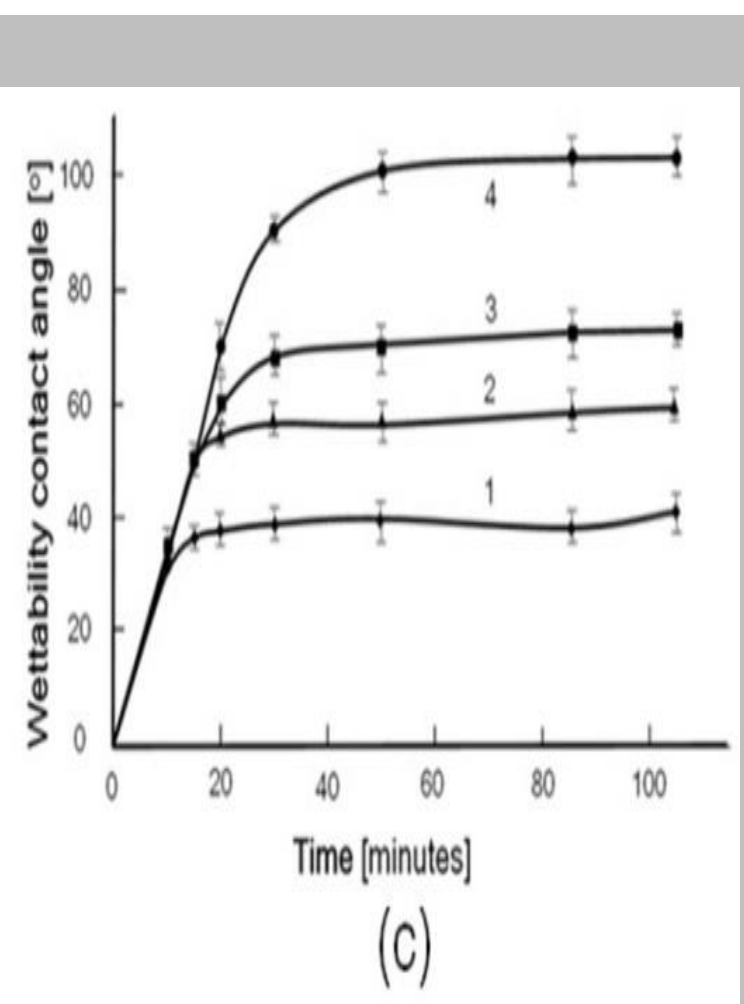

(C)

$\left(\mathbf{A}_{\mathbf{a}}\right)$

$\left(\mathbf{A}_{\mathbf{w}}\right)$

Fig. 2. (a) Superficial phospholipid bilayer of articular cartilage in water $\left(\mathbf{A}_{\mathbf{w}}\right)$ and (b) air-dry ( $\left.\mathbf{A}_{\mathbf{a}}\right)$ conditions. (c) Changes of the wettability contact angle as a function of air-drying time. Curve: (1) After a 19-min delipidization in (chloroform/methanol) $\left(2: 1\right.$, v/v), contact angle of 40 ${ }^{\circ}$; (2) after 9 min delipidization, contact angle of $63^{\circ}$, after 3 min delipidization, contact angle of $75^{\circ},(4)$ normal articular surface; contact angle of $104^{\circ}$. Contact angle $\mathrm{SD}(\mathrm{n}=5$, error bars $=95 \%$ confidence limit). 


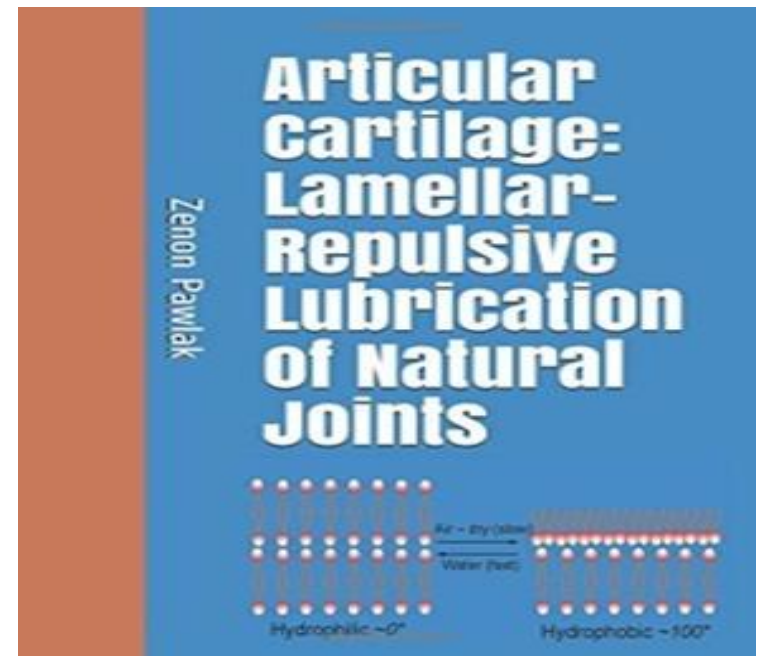

Fig. 3. Phospholipidic bilayers of articular cartilage under the wet and air-dry conditions. Variations in surface energy lead to conformational transformations in the surface phospholipids from bilayer (hydrophilic) to monolayer (hydrophobic). Book cover "Articular cartilage: Lamellarrepulsive lubrication of natural joints" [6].

The wetting studies on healthy cartilage and osteoarthritic surface in vivo indicate that, when exposed to dry atmospheric conditions, only hydrophobic chain is exposed to the outside. When the surfaces are becoming exposed to bulk water the surfaces rapidly become hydrophilic (Fig. 2). These findings are entirely consistent with those of the adhesion measurements $[7,8]$.

\section{Conclusions}

We studied how the wetting properties of the cartilage surface in vivo changed with dehydration. The results are described and summarized in
Fig. 2. It has been demonstrated that the "smart surface" of cartilage is super hydrophilic when wet and hydrophobic when air-dry, Fig.3. The key to understanding the mechanism of joint lubrication lies in obtaining an insight into the relationships between the structure and function of surface. The main result of the present study is the observation that the rate of lipid flip-flops is significantly enhanced by the spontaneous formation of water pores during dehydration. Evidently, one should then ask whether the rate of pore formation is comparable to the lipid flip-flop rate in the absence of pores. It is observed that lipid flip-flop is affected by dehydration activated processes.

\section{References}

1. Gurtovenko AA and Vattulainen I. Molecular mechanism for lipid flip-flops. J. Phys. Chem. B, V. 2007; 111, 48: 13557

2. Contreras FX, Sánchez-Magraner L, Alonso A, Goñi FM. Transbilayer (flip-flop) lipid motion and lipid scrambling in membranes. FEBS Lett. 2010; 584(9): 1779-86.

3. Structural Biochemistry/Lipids/Biological Membranes.

4. Hills BA (1988), The Biology of Surfactant.1988; London; Cambridge University Press.

5. Chappuis J, Sherman IA and Neumann AW (1983), Surface tension of animal cartilages it relates to friction in joints, Annals of Biomedical Engineering. 1983; 1: 435-449.

6. Pawlak Z (2018), Articular Cartilage: Lamellar-Repulsive Lubrication of Natural Joints, KDP, USA 161pp.

7. Leckband D, Chen You-Lung, Israelachvili J, Wickman HH, Fletcher $\mathrm{M}$ and Zimmerman R. Measurements of conformational changes during adhesion of lipid and protein (polylysine and S-layer) surface, Biotechnology and Bioengineering. 1993; 42:167-177.

8. Pawlak Z, Urbaniak W, Petelska AD, Yusuf, KQ and Oloyede A. Relationship between wettability and lubrication characteristics of the surfaces of contacting phospholipidsbased membranes, Cell Biochemistry and Biophysics. 2013; 65: $335-345$
This work is licensed under Creative Commons Attribution 4.0 License

To Submit Your Article Click Here: Submit Article

DOI: $10.31579 / 2690-1919 / 087$
Ready to submit your research? Choose Auctores and benefit from:

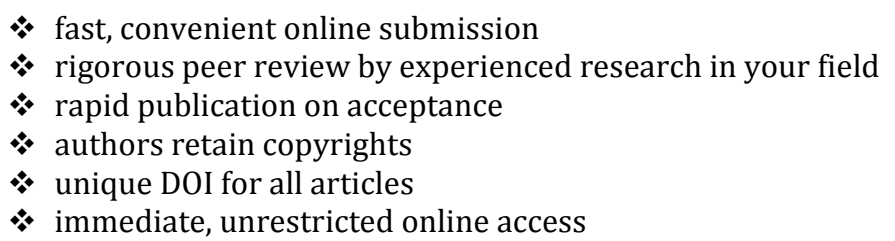

At Auctores, research is always in progress.

Learn more www.auctoresonline.org/journals/journal-of-clinicalresearch-and-reports 